\title{
The Tree of Knowledge System: A New Map for Big History
}

\author{
Gregg Henriques \\ James Madison University \\ Joseph Michalski \\ King's University College, Western Ontario \\ Steven Quackenbush \\ University of Maine at Farmington \\ Waldemar Schmidt \\ Oregon Health \& Sciences University
}

\begin{abstract}
This article summarizes the Tree of Knowledge (ToK) System (Henriques, 2003; 2011), and compares and contrasts its depiction of cosmic evolution as four "dimensions of existence" (i.e., Matter, Life, Mind and Culture) with Big History's eight thresholds of complexity. Both systems share the concern with the current fragmentation in academic knowledge and advocate for a more consilient and integrative vision that places the disciplines in coherent relationship to each other, and both views argue that such efforts are needed to advance wise decision making in the context of the accelerating rate of change. The major differences between the two perspectives are found in how the ToK conceptualizes the different dimensions of existence. Following Matter, the dimensions of Life, Mind and Culture are seen as emerging as a function of different semiotic or information processing systems that give rise to strongly emergent properties. In addition, given its emphasis on psychology and the mental dimension of existence, the ToK highlights some aspects of cosmic evolution that have not been featured prominently in most models of $\mathrm{BH}$. The article ultimately suggests that there is potential for a fruitful synergy between the historical emphasis of BH with the more psychological focus of the ToK System.
\end{abstract}

Correspondence | Gregg Henriques, henriqgx@jmu.edu; Joseph Michalski, jmichal2@uwo.ca; Steven Quackenbush, steven.quackenbush@maine.edu; Waldemar Schmidt,kokopelli@ccgmail.net

Citation | Henriques, G., Michalski, J., Quackenbush, S., Schmidt, W., (2019) The Tree of Knowledge System: A New Map for Big History. Journal of Big History, III(4); 1 - 17.

DOI | https://doi.org/10.22339/jbh.v3i4.3410

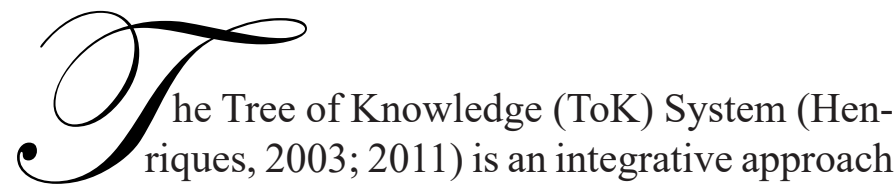

to scientific and humanistic knowledge that shares much in common with the Big History movement (Christian, 2017), even though the two visions were conceived of and developed independently. Central to the ToK System is a series of diagrams that offer a pictographic representation of the "unfolding wave of behavior" that has emerged since the Big Bang and has continued through the present. Because the ToK System is a representation of cosmic evolution and emergence on the dimensions of time and complexity, it is appropriate to characterize it as a Big His- tory $(\mathrm{BH})$ view of the universe, albeit a unique one. Indeed, it can be considered an explicit map of what some scholars in Big History have called "The Great Matrix" (Grassie, 2018). More specifically, the ToK System offers a new, systematic emergent naturalistic metaphysics (Cahoone, 2013) that defines key ontic concepts (i.e., Matter, Life, Mind, and Culture), and specifies their relations to one another and scientific knowledge about them. Figure 1 depicts the primary ToK System diagram (Henriques, 2003), and corresponds it with the eight Big History Thresholds.

As shown, the ToK characterizes the universe of behavior as consisting of four different dimensions of 


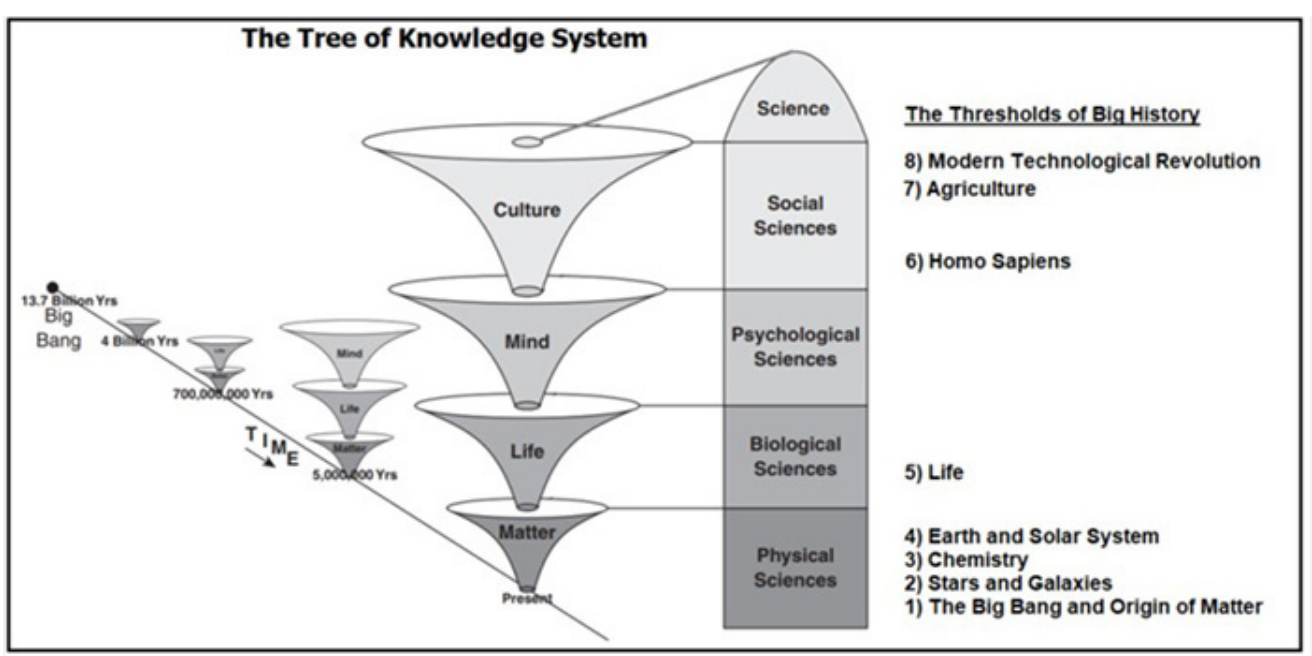

Figure 1. The Tree of Knowledge System and the Thresholds of Big History the theories, ideologies, and assumptions that guide the scientific enterprise, coordinate the behavior of scientists, and constitute scientific knowledge. In the language of the ToK, these conceptual ideological representations are characterized as "justification systems" (Henriques, 2011; Shaffer, 2008). Thus, the ToK depicts how the physical sciences map the dimension of Matter, the biological sciences map the dimension of Life, the existence. The "Matter" cone at the bottom represents the emergence and behavior of inanimate material objects from the time of the Big Bang, and includes entities such as atoms, stars, and planets. Particles like electrons represent the base of the cone as they are the simplest entities, whereas entities like macromolecules found in organic chemistry correspond to the top of the material cone. The "Life" cone represents the behavior of organisms, ranging from the simplest single-celled creatures (e.g., bacteria) up through large, complex multi-celled organisms (e.g., an oak tree). The "Mind" cone represents the behavior of animals with a brain, ranging from nematodes at the base (i.e., worms with simple brains) through highly complex and sophisticated animals, like chimpanzees, dolphins, and elephants. Finally, the "Culture" cone represents the behavior of human persons embedded in linguistic traditions and sociocultural historical contexts. It ranges in scale and complexity from individual persons to the behavior of modern, complex nation states or other societal structures organized by large-scale systems of justification (such as the United States of America).

As shown on the right side of the diagram, the ToK System includes the institution of science, which is depicted as emerging out of the dimension of Culture. What is specifically being represented are psychological sciences map the dimension of Mind, and the social sciences map the dimension of Culture. We can use the philosophical difference between ontology and ontic reality to achieve conceptual clarity in understanding this correspondence. As represented here, the sciences consist of the ontological knowledge of the actual, ontic entity. For example, the theory of general relativity represents the scientific, ontological understanding of how massive bodies curve space and time. As a theory about the material world, it resides in the matrix of the physical sciences, and thus is a part of the Cultural dimension of existence. However, the ontic behavior of the galaxies, having existed long before Einstein ever generated his theories about them, reside in the Matter dimension of complexity. It is the methods of empirical science and predictions and measurements of behavior that allow the two domains of ontological theory and ontic reality to be coherently connected.

\section{Correspondences between Big History and the ToK System}

The broad parallels between the ToK System and Big History (BH) are likely apparent to BH scholars. Advocates of both systems emphasize the point that to understand humanity, we must place it in an emergent evolutionary context. In direct alignment with $\mathrm{BH}$, the ToK System maps the universe on the 
axes of time (from past to present into the future) and complexity (from very simple elements present in the very earliest stages of the universe to increasingly complex entities, ultimately culminating in modern human societies). Moreover, both BH and the ToK System attempt to correspond the emergent domains of behavioral complexity in nature to the major areas of science (i.e., physics maps the most basic elements of nature, then chemistry, then biology, then the social or human sciences).

There is also significant alignment in the vision and values of both systems. In 2018, as the Executive Committee of the Theory of Knowledge (TOK) Society, we launched a conference series that sought to bring scholars together across the spectrum of academic knowledge and reflect on the "big" picture. Although the ToK System provides an anchor point for the TOK Society, it is also the case that we are interested in connections with other macro-level conceptions of knowledge and hence the broader name, "Theory of Knowledge," was used to denote our group. We embrace diversity of thought and the wide variety of different goals, perspectives, and topics that constitute the current academic structure. However, we also seek to anchor academic knowledge to a coherent overarching and organizing framework. In direct accordance with a primary mission of International Big History Association, the focus of the TOK Society centers on how the current fragmented pluralistic state of academic knowledge is problematic and might be reorganized into a more integrated pluralism. At the first TOK Society conference, scholars from philosophy, mathematics, biology, psychology, sociology, medicine and other disciplines offered perspectives that shared this goal and connected their visions to the Tree of Knowledge map of emergent evolution.

A second conference was held in April of 2019 that analyzed the ToK System in reference to the metaphysical and epistemological assumptions that shaped the Enlightenment thinkers and gave birth to the modern scientific enterprise. Consensus was achieved that the Enlightenment framework for science had produced a remarkably successful approach to the so-called "hard sciences," (e.g., physics, chemistry, biology). However, as E. O. Wilson (1998) notes in Consilience: The Unity of Knowledge, knowledge breaks down in its coherence as we move from biology into psychology and the social sciences. Consistent with the interdisciplinary, consilient vision of $\mathrm{BH}$, there was consensus that the ToK System provides a new metaphysical and epistemological framework that can potentially bridge this divide. The TOK Society thereby embraces the challenge of coherently organizing the complex interrelationships among the natural sciences, psychology, and the social sciences, while additionally specifying the vision and place of the humanities as key contributors as well.

It is important to note that these academic analyses were done as we reflected on the current backdrop of cultural and political unrest. Specifically, we consider the current age of "tragic mass shootings, a deeply troubled educational system, a broken political system that lacks intellectual integrity and produces excessive polarization and a large disenchanted political 'center,' global threats to democracies and the general world order, dramatic increases in mortality rates due to drugs and suicide, and skyrocketing rates of anxiety and depression in our youth," to be well-characterized as an "Age of Confusion" (see Henriques, Kroger, Michalski, Quackenbush, \& Schmidt, 2019). This sentiment and larger societal concerns align well with the vision of $\mathrm{BH}$, and its concern with both academic and cultural fragmentation and the disordered state of knowledge.

In the inaugural issue of the Journal of Big History, David Christian (2017) spelled out his vision of the BH movement and argued that $\mathrm{BH}$ "aspires to a universal understanding of history" (p. 12) where we are trying "to link the findings of specialist scholarship into a larger unifying vision" (p. 13). The first explicit goal he identified was that $\mathrm{BH}$ would empower us with a greater understanding of who we are and our place in the cosmos. The second goal was to offer a vision 
of existence that is both universal and consilient. Third, BH emphasizes the need for interdisciplinary collaboration and doing so via an integrative reference point that is not just located on "the individual islands and continents of modern scholarship," (p. 14) but includes a big picture frame that supports the many links between them. Finally, with an eye toward the dialectic between the sciences and humanities, Christian emphasized the point that $\mathrm{BH}$ can provide an "origin story" for the modern age, one that is grounded in science and can help humanity understand our place in the cosmos and how we might use that to chart a course toward a wiser future.

In a highly similar fashion, the ToK System has also been framed to offer a universal origin story for humanity and its place in the cosmos on the dimensions of time and complexity. It is named the Tree of Knowledge both because it views the nature of the universe as having its fundamental root beginnings as a primordial seed or atom, out of which the whole has flowered forth. The name is also a reference to the need to connect to the "mythos" of the past and develop meaning-making systems for the present. The metaphor further embraces the idea that we need the scientific curiosity and moral clarity that allows us to eat heartily from the Tree of Knowledge, and we can flourish in doing so. Moreover, in direct alignment with Christian's (2018) articulation of a need for an "origin story," we believe that "scientific knowledge does have a story to tell about humanity, and it is crucial that we convey such knowledge in the context of a meaningful narrative that explicitly emphasizes a moral component. The ToK System is a picture of the universe story, as presently mapped out by scientific inquiry that potentially provides us with a shared origin myth" (Henriques, p. 259, 2011). And, via the "fifth joint point" (Henriques, 2011), the ToK also orients us to consider the future and reflect on what Big History scholars call the Threshold 9 Big History Singularity that might emerge in the upcoming decades (Korotayev, 2018).

\section{Contrasts between the Big History Formulation and the ToK System}

Perhaps the easiest way to characterize the central differences between $\mathrm{BH}$ and the ToK System is to begin by recognizing the disciplinary home of the two originators. Whereas Dave Christian is a historian who specialized in world history, Gregg Henriques is a clinical psychologist who specializes in theoretical and philosophical psychology. Both shared the perspective that macro-level frames and perspectives were needed to effectively organize their fields and to situate disciplinary findings in a larger picture of understanding. Paralleling Christian's desire to situate the place of modern human history in the larger and more universal historical context, Henriques also sought to ground the field of psychology in a larger context of understanding (Henriques, 2003). However, as he attempted to do so, he realized that there was a profound "problem of psychology" (Henriques, 2008). This is the name he gave to the fact that the field of psychology lacked any consensually agreed upon definition or shared understanding of its subject matter. The ToK System is a metaphysical conception of the universe that we believe solves the problem of psychology's missing definition and ill-defined subject matter. As we will describe later, it does this by clearly delineating the "mental" dimension of existence, or what Cahoone (2013) calls the mental "order of nature." By identifying the mental with the animal and by separating out human psychology from a more basic psychology that includes all mental/ animal life, Henriques (2004) has argued that the ToK solves the problem of psychology and allows for a crisp definition of the field.

How the ToK functions to achieve this can be more clearly seen when we compare the ways $\mathrm{BH}$ and the ToK System divide the evolution of complexity. As noted, Big Historians use the term "thresholds" to describe the phases of emergent complexity. The eight thresholds include the following: 1) the Big Bang and Origins of Matter; 2) Stars and Galaxies; 3) Chemistry; 4) Earth and Solar System; 5) Life; 6) Homo Sapiens; 7) 
Agriculture; and 8) Modern Technological Revolution (Christian, 2018). These thresholds correspond to the disciplines of cosmology and particle physics, stellar and planetary astronomy, chemistry, geology, biology, and then the social sciences and related disciplines, like anthropology, archaeology, and history proper. BH's thresholds roughly correspond to- but are also different in important ways from - the ToK System's four primary dimensions of existence: 1) Matter; 2) Life; 3) Mind; and 4) Culture. As shown in Figure 1, the $\mathrm{BH}$ thresholds 1 through 4 correspond to Matter on the ToK System (and the subject matter of the "physical/ material sciences"), threshold 5 corresponds to Life (and subject matter of the "biological sciences"), and thresholds 6, 7, and 8 all correspond to Culture (and the subject matter of the "social sciences"). The difference between the eight thresholds and the four dimensions of existence is one key point of departure between the systems.

Noting the difference between the thresholds and the dimensions brings us to the second big difference between the ToK System and the Big History formulation. $\mathrm{BH}$ offers no identifiable place for the ontic domain of "Mind" and its corresponding science, what we call the "basic" science of psychology. The thresholds in BH jump from biology-which corresponds to the emergence of life approximately 3.8 billion years ago at threshold five - to the emergence of early modern humans studied by paleoanthropologists 250,000 years ago at threshold 6. At this point, the human social sciences (i.e., anthropology, archeology, history, sociology) become the prominent disciplines of inquiry. From our vantage point, this absence says more about the field of psychology than it does about $\mathrm{BH}$ scholars. The $\mathrm{BH}$ alignment provides a good illustration of how the boundaries of other disciplines (particle physics, chemistry, biology, even history) correspond to domains of inquiry that have at least vaguely identifiable boundaries. In contrast, as Henriques (2011) documents, psychology is a murky discipline that does not correspond to clear boundaries, but is vaguely positioned in the nebulous space between biology and the social sciences.

In the parlance of the ToK, basic psychology refers to "the mind, brain, and behavioral" sciences. Put simply, if we consider the mind to be what the brain does (as the cognitivists do) and consider what the brain does is to coordinate the behavior of the animalas-a-whole (as neuroscientists and behaviorists do), then basic psychology can be considered the science of the mental order of nature as delineated by the diagram. This conception of the science of psychology lines up with neo-behaviorist and cognitive science visions and includes the behavior of all animals with complex adaptive bodies, from fruit flies to squid to elephants (see Godfrey-Smith, 2016). Importantly, it is the dimension of existence when sentience (i.e., the capacity to experience the world via feelings) emerges. In his article, Psychology Defined, Henriques (2004) points out why human psychology is appropriately considered a special subset of basic psychology. This is because humans enter the dimension of Culture through language and become self-reflective entities that justify their actions on the social stage. This makes human behavior qualitatively different from the behavior of other animals. It should be noted that one of the points of confusion, both for psychology and for larger understanding, is that although the field of psychology's basic concepts are anchored to the mental, the vast majority of modern day psychologists emphasize the human individual. Henriques (2004) argues that this animal versus human confusion and misalignment is part of the problem of psychology.

Another important distinction between the models pertains to the nature of complexity. BH generally characterizes complexity as being on a single axis, one that goes from particles to atoms to molecules to organisms to societies, with "threshold shifts" in between. Consider that in $\mathrm{BH}$, the shift from particles and atoms that formed after the Big Bang into the stars reflects a threshold shift (i.e., from 1 to 2), as does the shift from chemistry to life (from 4 to 5). The ToK, however, recognizes two different kinds of emergence, 
one of which is "weak" and the other of which is "strong" (Clayton \& Davies, 2008). Weak emergence happens within the context of a dimension of existence. For example, the new properties that appear when molecules form from the bonding of atoms are considered weakly emergent properties. Although these are important and warrant the term "emergent", they do not represent a qualitative shift in the

\section{The Periodic Table of Behavior}

kind of behavioral complexity observed in the strongly emergent transitions. We will delineate in more detail later what we mean by "qualitatively" and "strong emergence".

One way that we have found helpful is to depict the map of complexity afforded by the ToK System in a "Periodic Table of Behavior." Behavior is defined here in the most general sense, which is change in objectfield relations over time. Different dimensions show different kinds of behavioral change patterns. The Periodic Table of Behavior (PTB) explicitly splits the analysis of complexity into two separate axes, giving rise to a new way to organize and classify behavior patterns in nature. One axis (the columns) consists of the four dimensions depicted by the ToK (i.e., Matter, Life, Mind, and Culture). The other axis (the rows) consists of the level of object-change analysis that is being considered (i.e., part, whole, or group) within each of the dimensions. When these two axes are differentiated, a clearer picture of the various kinds of behaviors that exist in nature emerges.

The PTB makes a distinction between a "general level of object field relations" and the primary or foundational units, with the former being listed above the latter. The general level of analysis refers to the various kinds of entities associated with that dimension of complexity (i.e., object, organism, animal and person), and the fields which such objects reside (i.e., field, ecology, environment, society). For example, a physicist might track the behavior of either an apple or a cannonball traveling through the four dimensional grid (i.e., the three dimensions of space, one of time). Both apples and cannonballs are "general" rather than "primary" physical. The PTB asserts that there are fundamental or "primary" whole units. Atoms are the primary whole units that operate in the material dimension, with subatomic particles being the parts and molecular levels and above being groups or clusters. Cells are the primary units that operate in the living dimension, animals with brains are the primary units for the mental dimension, and human persons for the cultural dimension.

With the Periodic Table explicitly mapping behavioral complexity by differentiating the dimensions from the levels of analysis, we can now move to explain more clearly how the dimensions of existence are characterized in the language of the ToK. This division of complexity into the four different 
dimensions of existence is one of the most novel features of the ToK and is central to understanding the metaphysical and ontological claims it makes.

\section{Matter: The First Dimension of Existence}

The first dimension of existence on the ToK System is called Matter. The technical definition of matter in the physical sciences is a material entity that has mass and takes up space. However, Matter on the ToK refers to something broader, namely it refers to the emergence of the ENERGY-MATTER-SPACE-TIME grid that is sparked at the "big beginning," (Wood, 2018) which is normally described as the Big Bang. Wood (2018) offers a powerful articulation of this dimension, as he helpfully delineated each of these concepts with an acronym that captures the essence of it. For example, Wood characterized "TIME" as "The Initial Moment of Emergence" and "ENERGY" as the "Entropic Nexus Emitting Radiation, Galaxies, and You". Consistent with Wood's analysis and modern cosmology, the ToK posits that the material dimension of existence emerged at the Big Bang (what Wood calls

\section{Matter: \\ The First Dimension of Existence}

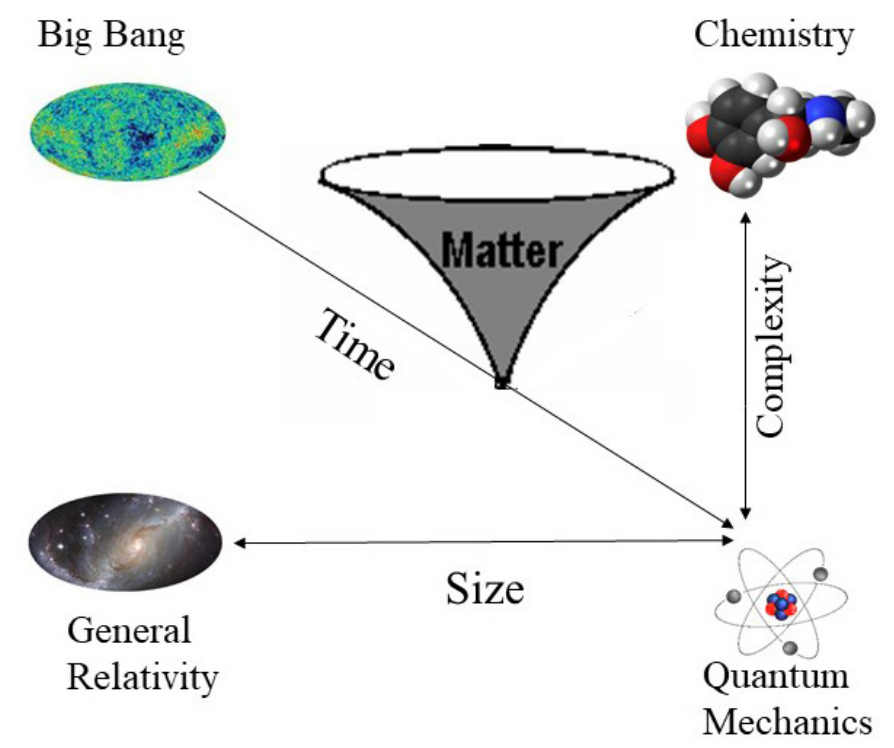

Figure 3. The Material Dimension of Existence. the "Big Beginning") and has grown in space, time and behavioral complexity since. Figure 3 depicts the key features of this dimension of complexity.

A word is necessary about the concepts associated with the Big Bang, which, as Wood (2018) notes, can be confusing and need to be updated in the context of modern inflationary models. Modern physicists debate exactly how the universe sprang into being from the "singularity." In technical terms, the word singularity is derived from Einstein's theory of general relativity and refers to a gravitational force of infinite density, resulting in the inward collapse of the dimensions of space and time on a single point. It is important to recognize that it is a mathematical concept rather than an empirically grounded, natural science one. There is significant uncertainty among physicists regarding the nature of the singularity or single, atom-like point at the "Big Beginning". For example, there are deep questions about whether it should be thought of as energy or as matter or as some novel combination of the two or some other entity, such as a quantum fluctuation in the spacetime void. The laws and concepts of physics break down at this point, which makes things especially murky at a metaphysical level of understanding.

Consistent with several theoretical physicists (Das, 2017), the ToK System characterizes the initial condition as a "pure energy singularity." This places energy as the most fundamental substance, and the ultimate common denominator in the universe. It needs to be acknowledged that this conception bends the standard definition of energy in physics, which is defined as capacity to do work. There are many different forms of energy that are interchangeable (e.g., gravitational, electromagnetic), but energy most commonly refers to the amount of an entity, as opposed to an entity per se. In this regard, "pure energy" is somewhat akin to saying "pure quantity"; thus, it is important to realize that the concept being applied is not crystal clear. However, all foundational concepts reach a point at which they become difficult 
to define or conceptualize, and energy is no exception. At bottom, energy is simultaneously a ubiquitous and abstract concept. As the physicist Richard Feynman (1963/1995, p.71) wrote, "we have no knowledge of what energy [fundamentally] is."

The idea that energy is foundational, even relative to matter, is a conception shared by many esteemed physicists. Consider this quote from Stephen Hawking (1988), who was speaking about the emergence of elementary particles from the Big Bang: "Where did [all the particles in the universe come from]? The answer is that, in quantum theory, particles can be created out of energy in the form of particle/antiparticle pairs" We can also think about the equivalence between energy and matter, as represented in Einstein's famous $\mathrm{E}=\mathrm{mc}^{2}$ formulation. Consistent with this formulation, many physicists, including Einstein, argued that matter could be fruitfully considered as being a form of frozen energy. In short, the ToK depicts Matter as a dimension of complexity that emerges out of a pure energy singularity at the time of the Big Bang.

Despite some uncertainty regarding the ultimate substance of the singularity, there is much agreement about what emerged immediately after the inflationary period and hot Big Bang. Within the first second following the initiation of the Big Bang, the energy singularity had divided into the familiar forms of forces and elemental particles that we see today. These include the four fundamental forces in nature (i.e., the electromagnetic, gravitational, and strong and weak nuclear forces) and the elementary material particles (e.g., quarks, which make up protons and neutrons, and leptons, a familiar example of which is the electron). The Standard Model of Elementary Particle Physics forms the base of the Matter dimension on the ToK.

We are now on much firmer conceptual footing and have the key ingredients for a scientific understanding of the basic processes or behaviors of the universe. Such ingredients include objects (i.e., particles and waves), fields, and the force interactions that take place between them, as well as change processes that occur on the dimensions of space and time. From this, we proceed to track the emergence of increasingly complex material objects. Just as Christian (2018) delineates in The Origin Story, as the early universe aged, it cooled and expanded, and electrons (negatively charged particles) and protons (positively charged particles) formed into simple atoms, like hydrogen and helium. Then, large gas clouds formed, which then began to collapse in on themselves as a function of gravity. This collapse resulted in the formation of stars. As time progressed further, increasingly complex material objects emerged, including entities such as planets. In addition, atomic elements like carbon, nitrogen, oxygen, phosphorous and sulfur were formed in the bellies of stars and when the stars exploded, they were launched out into the universe and then found their way to the surface of planets. Very complex chemical molecules formed on the surface of planets that allowed for their emergence (i.e., environments that were neither too hot nor too cold).

This emergence is characterized by $\mathrm{BH}$ as four different thresholds, represented by particle physics, astronomy, chemistry and geology. The ToK System acknowledges these as different kinds of behavior patterns and require different scientific language systems to map them. The behavior of plate tectonics is, after all, different from the behavior of electrons. However, according to the ToK, they all exist at the material dimension of complexity and are mapped by the various physical sciences. Cosmologists map the behavior of the universe, along with other macrolevel constellations like galaxies. Particle physicists map the behavior of subatomic entities like electrons. Chemists map the behavior of molecules, geologists the behavior of rock formations, and so forth. All of these are clustered together as the physical or material sciences precisely because they describe entities that behave at the Matter dimension. However, something even more different emerges when we shift into life. The biological sciences are not physical sciences. The reason, according to ToK metaphysics, is because "living" is a qualitatively different kind of behavioral pattern. 


\section{Life: The Second Dimension of Existence}

The second dimension of existence on the ToK System is called Life. Although it is highly probable that life exists elsewhere in the universe, the ToK System maps our current knowledge of the empirically documented universe. The best scientific evidence suggests that planet earth formed approximately 4.5 billion years ago. Life, in the form of simple single cells, was present on earth by 3.7 billion years ago and may have started as early as 4 billion years ago (Lane, 2016). Exactly how life originated remains a bit of a mystery, although there are many clues and several plausible models.

Biologists have long debated exactly what constitutes life. Living entities have several unique features that inanimate objects generally do not have. These properties include behaviors such as metabolism, homeostasis, adaptive responsiveness to the environment, growth, and reproduction. In 1944, the physicist Erwin Schrödinger authored a now classic book directly asking the question What is Life? Schrödinger pointed out what is remarkable about life is how it is organized, how it takes energy in to perform work to fend off entropy, and how it appears to be self-organizing. Another way of saying this is that living objects are remarkably complex and work to effectively and efficiently maintain that complexity. According to the ToK System, the best way to think about Life is to consider it as an emergent dimension of behavioral complexity. The key concept that gives rise to a new dimension is found in semiotics (Pattee \& Kull, 2009) and the interrelated concepts of sign translation, input-output information processing, computation, storage, communication and cybernetic feedback loops.

In the view provided by the ToK, the fundamental distinction between life and inanimate matter (particles, atoms, molecules, chunks of rocks, stars, etc.) is that the latter do not engage in information processing in the way that living organisms do. Information processing involves the following components: 1) inputs (data encoding), 2) data processing via computation, which includes storage and retrieval, 3) outputs (making changes based on inputs and computation), and 4) communication and feedback with other information processing units that 'speak the same language' (in this case, cell-cell communication). Information processing is crucial because, it gives rise to a separate dimension of causation. In chapter four of his book, Schrödinger stated that "living matter, while not eluding the "laws of physics" as established up to date, is likely to involve "other laws" hitherto unknown, which however, once they have been revealed, will form just as integral a part of science as the former". According to the ToK System, these "other laws" are to be found in the processes of biosemiotics (Pattee \& Kull, 2009), self-organization (Kaufman, 1995), negentrophic physiological first principles (Torday, 2019), and genetic information processing (Bray, 2009) and the overlap and interconnections between them.

It should be noted that inanimate entities can be fruitfully couched in terms of information. Indeed, we support Ken Solis' (2018) argument that Big History can be effectively described in terms of the "unfolding of information" that started at Time $=1$ Planck unit. The fact that physical systems can be framed in informational terms can also be seen in John Wheeler's phrase that we need to move "from it to bit" (Siegfried, 2000). However, when we are dealing with inanimate events, there are no data being processed, nor is there anything akin to an information processor, systematic computation, memory, outputs nor communication feedback systems that are tied to such processing occurring in inanimate objects or fields (Pattee \& Kull, 2009). Although a bouncing ball can be described in physical information terms, it behaves the way it does because of the current forces acting on it; there are no inputs being computed or referenced against a store of information, followed by output and communication with other entities. Figure 4 depicts the central features that characterize the Life dimension of existence. 


\section{Life: \\ The Second Dimension of Existence}

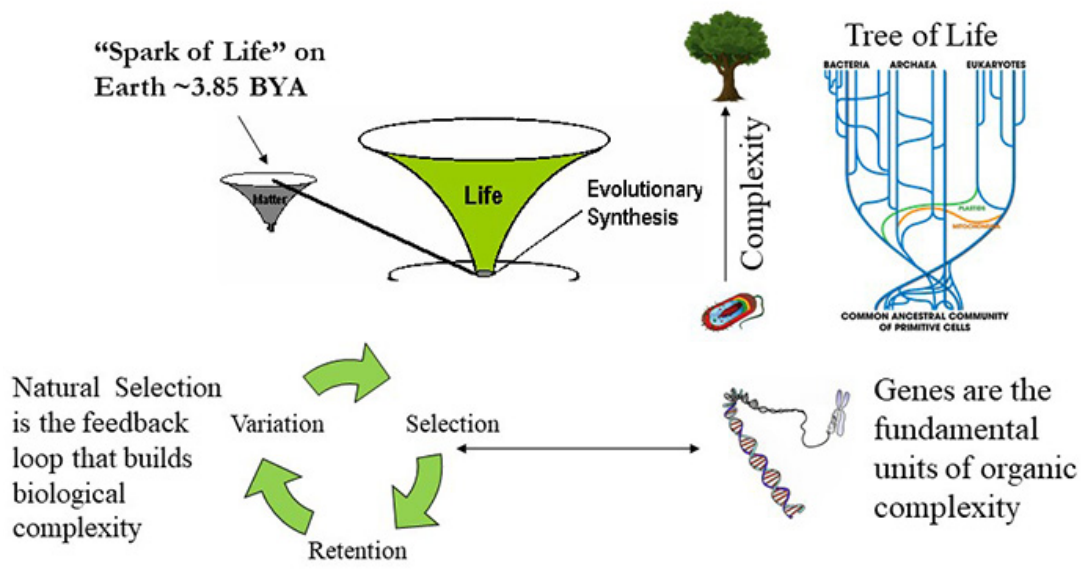

Figure 4. The Life Dimension of Existence.

In contrast to the inanimate world, life exists as a collection of information processing systems that have stored information across the generations and are shifting in response to ongoing experiences. As Dave Christian (2018, p. 79) put it, living organisms are "informavores" in that "they all consume information, the mechanisms they use for reading and responding" to their environments. This fact is present in the "language games" of biologists. Biologists speak of "the language of genetics" in that there are genetic messages, genetic software, and so forth. The famed DNA molecule is an information storage system, and the various RNA types (messenger, transfer, regulatory etc.) work as transformational entities that take the information encoded in the DNA and translate them to allow for the formation of proteins.

Many biologists have articulated in detail the utility of thinking about life in terms of information processing. In Wetware: A Computer in Every Living Cell, Bray (2009) articulated how the DNA and RNA complexes function as computational systems that give life its complexity. Farnsworth, Nelson and Gershenson (2012) go further and argue that the defining feature of life is information processing, and that it not only resides in the DNA and RNA molecular structure, but functional information processing is also woven together at all levels of life, from the genetic to the cellular to the ecological. They argue it is the central concept that allows biologist to understand the unique organized features and properties of living entities. The key point here is that living matter behaves qualitatively different from inanimate matter, and both the language of and properties associated with information processing are the root of this qualitative difference.

Genes can be considered the fundamental unit of information in the Life dimension of existence. The fundamental structural whole that allows the component parts to engage in information processing, along with metabolism, growth and reproduction, is the cell. As a fundamental unit, the cell is to Life what the atom is to Matter. For over a billion years, cellular life maintained a relatively basic structure (Lane, 2016). Then, at about 2 billion years ago, a massively important structural change happened when there was a remarkable jump in cellular complexity. That jump was the emergence of eukaryotic cells, meaning cells that had a nucleus contained in a membrane. Eukaryotic cells were a game changer in terms of behavioral complexity at the biological dimension. Such cells are much larger and far more structurally complex than simple cells like bacteria. Even more important, they set the stage for the emergence of multi-celled creatures, what Christian (2018) calls "Big Life," which is a "sub-threshold" in the BH system. Multi-celled creatures like plants exhibit many emergent properties that are not present in single-celled creatures. From a ToK perspective, the shift from cells to plants is similar in kind to the shift from atoms to molecules. The interaction of the parts does indeed create emergent properties. Although they are "higher up" on the Life cone in their degrees of behavioral complexity, plants and fungi remain at the dimension of Life because their behaviors are 
mediated by bio-information processing systems.

However, in one kingdom some multi-celled creatures did make a qualitative jump in behavioral complexity, and they did so in a manner that paralleled the shift that came with the emergence of life itself. Whereas the cell became a genetic information processing system that allowed for a new dimension of behavioral complexity, approximately a half-billion years ago a novel information processing system emerged that coordinated the multiplicity of cells. This resulted in a large organism that had the capacity to sense its environment, coordinate complex bodily movements, and respond to immediate feedback as a whole entity.

\section{Mind: The Third Dimension of Existence}

The third dimension of complexity on the ToK System is called "Mind." Mind is a term that has many different meanings in common parlance. It sometimes refers to thoughts or cognition, sometimes to sentience (the experience of subjective feelings) sometimes to self-awareness (reflection on feelings) and sometimes to observable actions (as in, "We saw him put his mind to that task"). Others equate it to the brain. In the ToK language system, when capitalized, "Mind"

\section{Mind: \\ The Third Dimension of Existence}

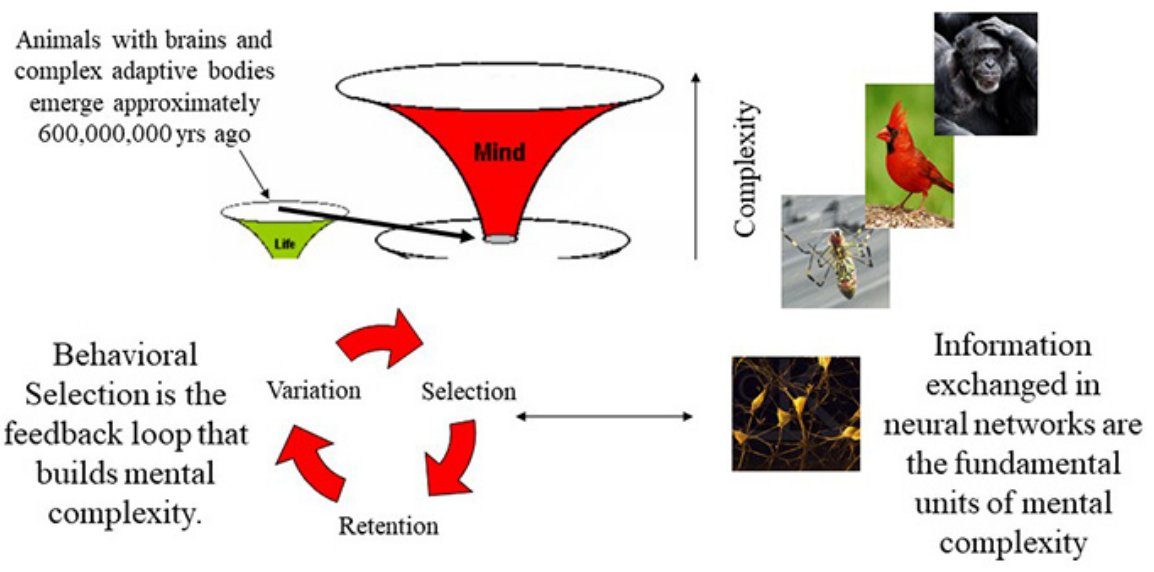

Figure 5. The Mind Dimension of Existence is like Matter, Life, and Culture in that it refers to an identifiable dimension of existence in nature. Whereas Matter corresponds to the behavior of objects, Life to the behavior of organisms, Mind corresponds to the behavior of animals, especially those with "complex adaptive bodies".

Figure 5 depicts the key defining features of Mind as the third dimension of existence on the ToK. It is arranged in a way that parallels the depictions offered for Matter and Life. The core logic for differentiating the behavior of animals with brains from other organisms (i.e., cells, plants, and fungi) is the same as the logic differentiating organisms from inanimate objects. The brain and neural networks are to an animal what DNA and genes are to a cell: a centralized, information relay and storage system. Just as genetic/ epigenetic information processing networks link molecules together to form a qualitatively different dimension of complexity, brains link cells together to form an animal whole that can behave as singular units that exist in a higher dimension of complexity. Just as biosemiotics are required for understanding life (Pattee \& Kull, 2009), cognitive neuroscience is the equivalent of a "neuro-semiotics" that is required for understanding the biological to psychological informational transition point (Tryon, 2016).

Mind is a dimension that is central to psychology, for it is the dimension where all the basic (i.e., nonhuman) mental properties emerge. Specifically, as animals with brains evolved into increasingly complex forms, we see a flowering of new capacities, such as learning, feeling and thinking. Animals are multicellular creatures that move around in their environment. They are also heterotrophic, meaning that they rely on other organisms for their energy sources (i.e., they need to eat other organisms because they cannot transform the energy of the sun directly into workable forms). The elements of 
free movement combined with the requirement of finding and eating other organisms were the central forces that shaped the structure and function of the nervous system - the centralized information processing system that allows for the coordination of the behavior of the animal as a singular unit. The evidence for the earliest emergence of animals with the beginnings of a nervous system points to their appearance approximately 650 million years ago. Such animals were similar in form and function to modern day jellyfish. They did not have brains, but had only distributed neural networks.

As Christian (2018) notes, a remarkable transformation happened during the Cambrian Explosion approximately 550 million years ago. This is when animals with brains emerged and began to dominate the landscape. Often referred to as "the organ of behavior," the primary task of the nervous system in general and brain in particular is to guide the animal to approach energy sources that enhance survival and reproduction (i.e., prey, mates, enriching territories) and avoid sources that are destructive (i.e., predators, toxins, degraded territories). Explicitly drawing a clear dividing line between the behaviors of animals and other organisms is a key and novel feature of the ToK System. It highlights unequivocally that animals with brains represent a qualitatively different kind of entity.

Moving from invertebrates like worms and butterflies into vertebrates like fish and reptiles and finally into mammals and primates, we can see that there has been an evolution of the nervous system and mental behavioral complexity. The task of the basic psychological sciences (or the mind, brain, animal behavior sciences) is to map this dimension in all its complexity. The most basic of these phenomena include neuro-motor reflexes, and habituation and sensitization, the earliest forms of animal learning. Then there are more complex, instinctual fixed action patterns. Then operant behavior patterns emerge, ones that are much more flexible to being shaped by consequences. Presumably, this is where the earliest forms of consciousness or sentience emerged, as experiences of pleasure and pain guide animals toward and away from environmental stimuli (GodfreySmith, 2016). Then, more complex emotions and intimate social relations appear, including attachment and affiliation, status seeking, and complex problemsolving indicative of imaginative thought, and much more.

In sum, when capitalized, Mind refers to the third dimension of existence and consists of the set of mental behaviors. Mental behaviors correspond to the behavior of animals with a nervous system. The adjective mental refers to that which makes the behavior of animals so different from atoms and cells. In the ToK language system, mental behaviors are the proper subject matter of the basic science of psychology. However, ToK System also shows us that the behavior of people (represented by the dimension of Culture) is different from the behavior of animals. This straddling of two dimensions of existence (i.e., Mind/animal and Culture/person) is central to understanding psychology's problems with its subject matter. The behaviorists were primarily interested in animal behavior. This was in direct contrast with the introspectionists like Titchener, who argued that psychology's proper subject matter was human perceptual consciousness analyzed in the lab. The ToK argues that we need to split psychology into two domains, basic and human, with the former corresponding to animals in general and the latter to humans at the individual level. The relationship between the two domains can be clarified when we consider the following remark from Tolman in his 1937 APA Presidential Address, when he stated:

[E]verything important in psychology (except such matters as the building up of a super-ego, that is everything save such matters as involve society and words) can be investigated in essence through the continued experimentation and theoretical analysis of the determiners of rat behavior at a choice point in a maze. (1938/1978, p. 364)

In other words, psychologists are interested in the 
drivers of animal behavior at its base, and, in addition, the field is concerned with human behavior at the individual level, which is distinctive from nonhuman animals because, as Tolman alludes, it takes place within a larger socio-linguistic context. This is why human psychology is so different from basic psychology, and it is to that context that we now turn.

\section{Culture: The Fourth Dimension of Existence}

The behavior of self-conscious people in sociolinguistic contexts resides in the dimension of existence called Culture on the ToK. The logic of why it is a separate dimension of existence parallels the argument given for the strong emergence of Life and Mind. Culture emerges because of a new semiotic system, human language. Human language is an open, symbolic syntactical information processing system that connects minds together to give rise to human societies. As Christian points out (2018), this is transformative in part because human language allows for collective learning, which is the sharing of information cheaply and effectively, and in a way that does not require direct experience. Although other animals have sophisticated systems of communication,

\section{Culture:}

\section{The Fourth Dimension of Existence}

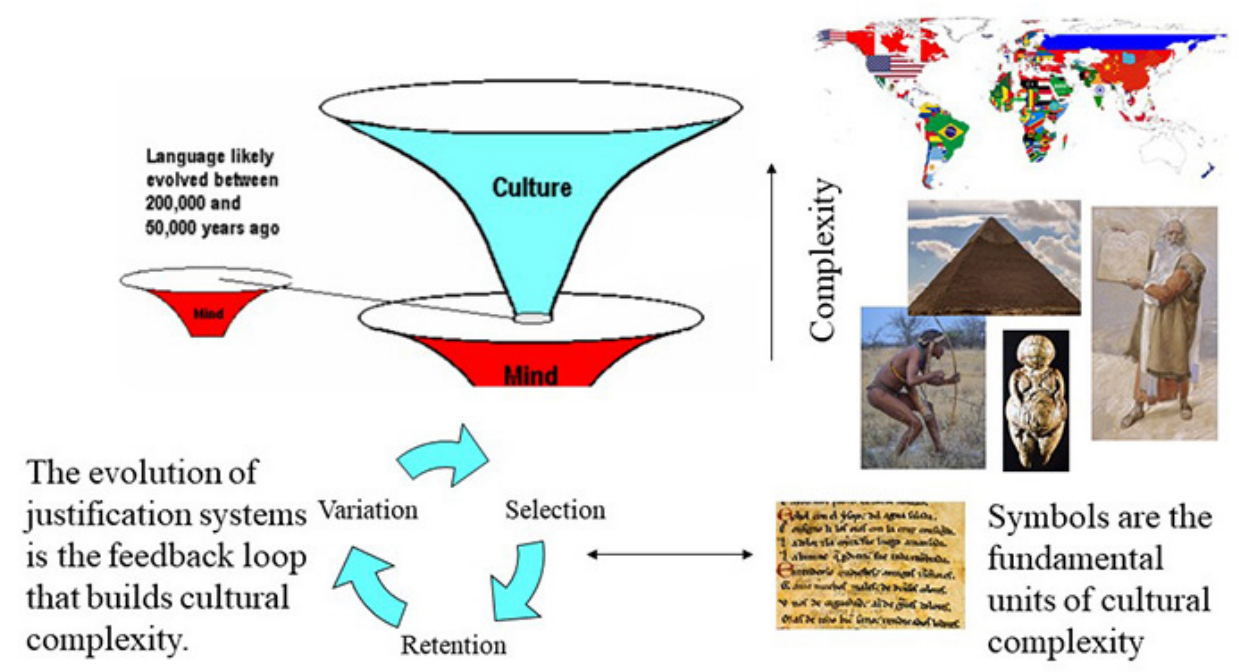

Figure 6. The Cultural Dimension of Existence human language is unique. It is an open communication system that includes learned symbols, grammatical syntax, and semantic meaning. Figure 6 offers a depiction of the dimension of Culture, which has clear parallels to the other dimensions of complexity.

The ToK System specifically characterizes Culture as large-scale systems of justification (Shaffer 2008). Justification systems refer to interlocking networks of linguistically represented beliefs and values that coordinate human action by framing both what is and what ought to be. As such, justification systems represent how shared beliefs and values functionally define, influence, and coordinate the human social field. Justification systems can range from the individual level (when a person talks privately to herself) to the dyadic level (a conversation) to the group level (e.g., as when preacher gives a Sunday sermon), and ultimately to the large-scale level of nations, political, or religious systems (e.g., the American legal system, a religion like Christianity, or the institution of science). The large-scale systems of justification are the essence of Culture in that they provide the macro-level contexts for justification. Such a formulation allows us to assert that the essential difference between a person and other animals stems from the former's capacity as an entity who deliberatively navigates the world of justification systems, both privately (to one's self) and publicly (to others).

The ToK offers a clear perspective on the evolution of Culture. It posits that between 200,000 and 50,000 years ago a number of different forces converged to transform a fractured mimetic sign system (see Donald, 1991) of communication into an open language system that enabled full-throated "question and answer" dialogue. The ToK System posits that the linguistic capacity to ask questions resulted in a new 
and powerful evolutionary adaptive problem. This is called the problem of social justification, and it refers to explaining to others the reasons for one's actions. The ToK posits this created a tipping point and feedback loop that gave rise to the Cultural dimension of existence. Anyone who has raised a child knows that children first learn simple commands and descriptions for objects (e.g., no, mommy, juice). After they achieve some command of descriptive language, a transformation happens, usually around the age of two. This is when they start asking questions. Because there are many ways in which individual development replays evolutionary history, we can make the claim that there were periods of concrete descriptive language that then evolved into more abstract question and answer process. Why? Because, although questioning is relatively easy, answering questions raises a whole new series of problems. Spending time with an intelligent, curious four-yearold makes this clear: "Why don't we eat cookies before we eat dinner?"; "Why are you bald?"; "Why is the sky blue?" As such children readily demonstrate, asking questions is much easier than answering them. That is why exasperated parents eventually say: "That is just the way it is!"

Processes of social justification creates a shared social epistemology, which refers to the intersubjectively shared and linguistically mediated social construction of knowledge. It is characgterized as the "mythic" phase in human consciousness and culture by Merlin Donald (1991). We connect this to the insights of Berger and Luckman (1957), in their work, The Social Construction of Reality, in that the emergence of social epistemologies is central to what makes human consciousness and behavior so unique in the animal kingdom. Such cultural processes were likely in full force by 50,000 years ago.

It is important to note that the evolution systems of justification provides a clear reason as to what gave rise to the search for the transcendent. The explanation is that as people develop the capacity to ask "why" there is a need and longing to anchor the answers into larger meaning-making systems. The need to ground and embody such meaning-making systems likely is what drove the more recent construction of scare temples, like that found at Göbekli Tepe (Aslan, 2018). A brief summary of the sequence from there to modern history can now be traced. The Temples allow for the alignment of investment practices, technology and meaning making. They require more centralized ways of being and that gives rise to the need for agriculture. That creates more centralized and complex social arrangements, and this necessitates more sophisticated record keeping. This drives the emergence of writing. Writing is external memory and marks the beginning of the shift from pre-modern mythic to modern Culture. An important reason for the transition is that writing sets the stage for systematic quantification, external memory and formal "history".

Twenty-five hundred years ago, there was a general large-scale emergence of what we consider to be more "formal" systems of justification. Such systems likely relate to the appearance of writing and elite literacy. Labeled by some the Axial Age (Eisenstadt, 1986), formal justification systems that we can label "philosophy" begin to emerge at various places in both the East and West during this period. In the ancient Western intellectual tradition, Socrates builds off the work of the Pythagoreans and others and gives birth to formal modes of epistemological inquiry. Via systematic questioning likely with mathematics as the ideal representation of authentic knowledge, Socrates realizes that social epistemology is (potentially) vacuous, hence his famous claim that he is wise only in the sense that he knows that he knows virtually nothing. Plato and Aristotle take up the mantle to develop formal-analytical philosophies that withstand Socratic-like philosophical criticism. This is the beginning of formal, refined academic knowledge as separate from commonsense social epistemology (at least in the West). In the $15^{\text {th }}$ and $16^{\text {th }}$ centuries, natural philosophy grew into modern science. The reliance on empirical methods and the mathematical mapping of matter in motion as achieved by Newton gives rise to the Enlightenment dream of purely rational justification. This gives rise to the modern institution of science, which has evolved out of Culture and functions to map the Big History of the universe and 
our place in it.

\section{The Fifth Joint Point, the Singularity, and the Future} of Humanity

As Korotayev (2018) notes, a number of futurists and scholars associated with Big History have noted a striking pattern associated with the acceleration of complexity over time. Made perhaps most wellknown by Ray Kurzweil's (2005) The Singularity is Near, the acceleration is such that it provides a clear "Singularity Hypothesis" in the Big History landscape, and a number of scholars have argued that "Big History Threshold 9" should appear between 2027 and 2045. Offering an in-depth review of several similar models of the accelerating evolution of complexity, Korotayev (2018) concludes that there are significant data "to indicate the existence of sufficiently rigorous global macroevolutionary regularities (describing the evolution of complexity on our planet for a few billion of years), which can be surprisingly accurately described by extremely simple mathematical functions." However, he does not believe that this "singularity" event will be necessarily transformative. Rather, he believes there are more reasonable interpretations that frame "this point as an indication of an inflection point, after which the pace of global evolution will begin to slow down systematically in the long term".

The ToK offers an interesting perspective on the concept of a "singularity" appearing in the relatively near future. We can start by noting that the ToK includes the insight regarding the accelerating pace of complexity and change. What it suggests, further, is a way to frame it. Specifically, the ToK suggests the possibility of the emergence of a new dimension of existence in a way that overlaps with the timeframe specified by Korotayev (2018). The ToK posits that novel dimensions of existence occur when new information processing or semiotic systems emerge and then become networked together and regulated via a centralized control system. These transitions are called "joint points" in the ToK language system, and the ToK identifies joint points between Energy and Matter, Matter and Life, Life and Mind, and Mind and
Culture (Henriques, 2011).

As noted, each dimension of existence following Matter has been associated with a new "semiotic" system (e.g., genetics, neuronal information processing, human language). Based on this logic, we can ask: Are we seeing the emergence of novel information processing systems and have they become networked together in a centralized way? There are some obvious technologies that present themselves in a way that results in us answering in the affirmative. Computers, the internet, and the interface between human and artificial intelligence systems are highly suggestive of an answer that might be "yes".

In accordance with predictions made by the futurist philosopher Oliver Reiser over fifty years ago (in 1958), there does appear to be a "world sensorium" that is emerging based on new information processing, and this is, perhaps, a reasonable interpretation of the singularity that has been identified by so many futurists. In the language of the ToK, this is known as "the fifth joint point" (Henriques, 2011). It is the possibility that we are on the cusp of a new emergence, that of a metamodern, Meta-Cultural dimension of existence.

The ultimate vision we embrace is that we need to wisely merge our knowledge of the cosmos and our world, our human natures, and the nature of human societies, and emergent technologies and artificial intelligences with foundational, universal values that enable core considerations regarding human dignity, well-being and integrity. Put another way, with its scientific humanistic philosophy, the ToK System orients us toward the crucial question: Given the remarkable pace of change in our world, can we foster the growth of environments and ways of being that enhance dignity and well-being with integrity? (Henriques, 2011).

\section{Conclusion}

In sum, the ToK System represents a new map of cosmic evolution. Consistent with many models that adopt a Big History (e.g., Chaisson, 2005) or related emergent naturalistic perspectives (e.g., Cahoone, 2013), the ToK System depicts the grandeur of cosmic evolution on the dimensions of time and complexity. 
What makes the ToK unique, however, is that it explicitly characterizes the universe as an unfolding wave of energy and information that has produced four distinguishable dimensions of existence, defined as Matter, Life, Mind, and Culture. The latter three dimensions of behavioral complexity are explained as the emergent functional consequences of distinct semiotic or information processing systems (i.e., genetic, neuronal, and linguistic).

The similarities and differences between the $\mathrm{BH}$ movement and the ToK afford scholars many interesting points of analysis. Most obviously, the correspondence and relation between the eight thresholds of $\mathrm{BH}$ and the dimensions of existence on the ToK warrants deep consideration. In particular, the ToK suggests that a threshold titled "Mind" should be added between Big Life and the appearance of humans. Finally, it should be noted with encouragement that both $\mathrm{BH}$ and the ToK situate humans in the cosmos in an effort to foster greater understanding of the past with the goal of charting a wiser course in the future. Given the current state of confusion and fragmented pluralism, it is affirming to see this convergence of viewpoints.

\section{References}

Aslan, R. (2018). God: A human history. Random house.

Berger, P. L. \& Luckman, T. (1966). The social construction of reality: A treatise in the sociology of knowledge, Garden City, NY: Anchor Books.

Bray, D. (2009). Wetware: A Computer in Every Living Cell, New Haven: Yale University Press.

Cahoone, L. (2013). Orders of nature. New York: Stony Brook Press.

Chaisson, E. (2005). Epic of evolution: Seven ages of the cosmos. New York: Columbia University Press.

Christian, D. (2017). What is Big History? The Journal of Big History, https://doi.org/10.22339/jbh.v1i1.2241 1, 4-19.

Christian, D. (2018). Origin story: The big history of everything. New York: Little, Brown and Co.

Clayton, P., \& Davies, P. (2008). The re-emergence of emergence: The emergentist hypothesis from science to religion. Oxford University Press.

Das, T. (2017). Origin of singularity in big bang theory from zero point energy. Canadian Journal of Physics, 95, 767-769.

Donald, M. (1991). Origins of the Modern Mind: Three Stages in the Evolution of Culture and Cognition. Harvard University Press.

Eisenstadt, S. N. (1986). The origins and diversity of Axial Age civilizations. SUNY Press.

Farnsworth, K, D., Nelson, J., \& Gershenson, C. (2013). Living is information processing: From molecules to global systems. Acta Biotheoretica, 61, 1-25.

Feynman, R. (1963/1995). Six easy pieces: Essentials of physics explained by its most brilliant teacher. California: Perseus Books.

Godfrey-Smith, P. (2018). Other minds: The octopus, the sea, and the deep origins of consciousness. William Collins.

Grassie, W. (2018). The great matrix of all beings. Journal of Big History, https://doi.org/10.22339/jbh.v2i2.2301 


\section{2(2), $37-52$.}

Hawking, S. (1988). A brief history of time: From the big bang to black holes. London: Bantam Books.

Henriques, G. R. (2003). The Tree of Knowledge System and the theoretical unification of psychology. Review of General Psychology, 7, 150-182.

Henriques, G. R. (2004). Psychology defined. Journal of Clinical Psychology, 60, 1207-1221.

Henriques, G. (2008). The problem of psychology and the integration of human knowledge: Contrasting Wilson's Consilience with the Tree of Knowledge System. Theory and Psychology, 18, 731-755.

Henriques, G. R. (2011). A new unified theory of psychology. New York: Springer.

Henriques, G. R., Kroger, E., Michalski, J., Quackenbush, S., \& Schmidt, W. (2019). Toward an Age of Clarity. Psychology Today. Blog post, March 25, 2019, retrieved from https://www.psychologytoday.com/us/ blog/theory-knowledge/201903/toward-the-age-clarity.

Kauffman, S. (1995). At home in the universe: The search for laws of self-organization and complexity. London: Oxford University Press

Korotayev, A. (2018). The $21^{\text {st }}$ Century Singularity and its Big History Implications: A re-analysis. Journal of Big History, https://doi.org/10.22339/jbh.v2i3.2329 2(3), 74-120.

Kurzweil, R. (2005). The singularity is near: When humans transcend biology. New York: Penguin.

Lane, N. (2016). The vital question: Why is life the way it is? Profile Books

Pattee, H. H. \& Kalevi K. (2009). A biosemiotic conversation: Between physics and semiotics. Sign Systems Studies, 37(1/2), 311-331.

Reiser, O. L. (1958). The integration of human knowledge. Boston: Porter Sargent.

Schrödinger, E. (1944/1967). What is life? The physical aspect of the living cell and mind and matter. Cambridge: University Press.
Shaffer, L. S. (2008). Religion as a large-scale justification system: Does the justification hypothesis explain animistic attribution? Theory \& Psychology, 18, 779799. doi:10.1177/0959354308097257.

Solis, K. (2018). The unfolding of "information" in Big History. Journal of Big History, https://doi. org/10.22339/jbh.v2i1.2254 2(1), 43-62.

Torday, J. (2019). Evolution: The "mechanism" of Big History - The grand synthesis. Journal of Big History, https://doi.org/10.22339/jbh.v3i2.3220 (3)2, 19-24.

Tolman, E.C. (1938/1978). The determiners of behavior at a choice point. In E.R. Hilgard (Ed.), American psychology in historical perspective (pp. 337-370). Washington, DC: American Psychological Association. (Original work published 1938).

Tryon, W. (2016). Transtheoretic transdiagnostic psychotherapy. Journal of Psychotherapy Integration, 26(3), 273-287. http://dx.doi.org/10.1037/a0040041

Wilson, E. O. (1998). Consilience: The unity of knowledge. New York: Alfred A. Knopf, Inc.

Wood, B. (2018). Imagining the unimaginable: Narratives of the Big Bang, Time, Space, Matter, Energy. Journal of Big History, https://doi.org/10.22339/jbh.v2i1.2251 2(1), 2-13. 
